\title{
CARACTERIZACÃO DE PASTAS MINERAIS PRODUZIDAS COM LAMAS DE REJEITOS METÁLICOS E NÃO METÁLICOS
}

\author{
R. H. O. BARREDA ${ }^{1}$, G. E. S. VALADÃO ${ }^{2}$ \\ Universidad Arturo Prat ${ }^{1}$, Universidade Federal de Minas Gerais ${ }^{2}$ \\ rolcay@gmail.com ${ }^{1}$
}

Submetido em 09/12/2019 - aceito em 23/03/2020

DOI: $10.15628 /$ holos.2020.9254

\section{RESUMO}

A disposição de rejeitos como pasta possui várias vantagens em comparação às disposições convencionais, por exemplo: utiliza uma área menor, melhora a recuperação de água, maior segurança operacional das barragens, etc. Neste trabalho foram caracterizadas as pastas preparadas com rejeitos de manganês e fosfato. $\mathrm{Na}$ caracterização dos sólidos foram determinadas: análise granulométrica, determinação do peso específico e identificação das principais espécies mineralógicas. Nas pastas foram determinadas: altura de abatimento, ângulo de repouso (preliminar) e algumas propriedades reológicas (viscosidade e tensão de escoamento). Os principais resultados foram: i) Espécies mineralógicas predominantes no rejeito manganês (RM): Pirolusita e Caulinita, no rejeito fosfático (RF): Calcita e Dolomita; ii) O tamanho médio dos sólidos foram 6,23 $\mu$ m (RM) e 4,76 $\mu \mathrm{m}$ (RF); iii) O RM e RF apresentam características de pasta desde $50 \%$ e $70 \%$ sólidos em massa respectivamente; iv) Foram atingidos ângulos de repouso superiores a $8^{\circ}$ (RM) e $16^{\circ}$ (RF); v) A viscosidade do RF foram superiores que o RM; vi) As tensões de escoamento do RM e RF aumentam exponencialmente desde $45 \%$ e $65 \%$ sólidos em massa respectivamente.

PALAVRAS-CHAVE: Pasta, Reologia, Disposição, Rejeitos, Barragens.

\section{CHARACTERIZATION OF MINERALS PASTES PRODUCED WITH SLUDGES OF METALLICS AND NON METALLICS TAILINGS}

\begin{abstract}
The disposal of tailings in the form of paste has several advantages compared to disposal conventional, for example: use a less area, improved recovery of water, greater operational safety of the dams, etc. In this work was characterized the pastes prepared with manganese and phosphate tailings. In the characterization of the solids were determined: granulometric analysis, determination of specific weight and identification of the main mineralogical species. In the pastes were determined: height of slump, repose angle (preliminary) and some rheological properties (viscosity and yield
\end{abstract}

stress). The main results were: i) Predominant mineralogical species in manganese tailings (MT): Pyrolusite and Kaolinite, in phosphate tailings (PT): Calcite and Dolomite; ii) Average solids size were $6.23 \mu \mathrm{m}$

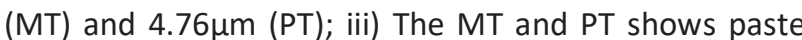
characteristics since $50 \%$ and $70 \%$ solids in mass respectively; iv) Were reached angles of repose above $8^{\circ}$ (MT) and $16^{\circ}$ (PT); v) The viscosity of pastes PT were higher than MT; vi) The yield stress of MT and PT increase exponentially since $45 \%$ and $65 \%$ solids in mass respectively.

KEYWORDS: Paste, Rheology, Disposal, Tailings, Dams. 


\section{INTRODUÇÃO}

As atividades de mineração vêm gerando ao longo dos anos consideráveis quantidades de rejeitos, sendo normalmente não reaproveitáveis, transformando-se assim em passivos ambientais. A disposição de rejeitos mediante o método convencional em barragens apresenta riscos operacionais e socioambientais. O estudo de alternativas para disposição e/ou reaproveitamento dos rejeitos torna-se necessário. As técnicas de espessamento, filtragem e centrifugação vêm se apresentando como atraentes para o desaguamento de rejeitos.

De acordo com Qi et al. (2011), a lama é um sistema coloidal no qual as pequenas partículas formam uma suspensão estável na água sendo difícil a separação destas da fase líquida. Segundo Paterson et al. (2004), as pastas minerais são polpas com elevadas concentrações de sólidos que são geralmente produzidas através da combinação de espessadores, filtros, ciclones e misturadores. As pastas minerais apresentam usualmente uma certa quantidade de finos (pelo menos $15 \%<20 \mu \mathrm{m}$ ) e suas características reológicas são, em geral, não newtonianas.

A disposição de rejeitos como pasta mineral é uma alternativa tecnológica que apresenta diversas vantagens como uma menor área para disposição, uma melhor recuperação de água, maior estabilidade do material disposto entre outros. Segundo Fernandez et al. (2013), os rejeitos espessados e pasta são mais uma opção para o tratamento e disposição de rejeitos.

Recentemente espessadores de pasta foram desenvolvidos para produzir elevadas concentrações de sólidos na descarga do equipamento (underflow) aumentando assim também a quantidade de água que pode ser recuperada do processo de forma imediata.

Considerando-se as características das pastas minerais, estudos reológicos são importantes para a verificação das características de escoamento em cada situação. Segundo Summerhaysa e Gaspar (2016), a tensão de escoamento é definida como o esforço que deve ser aplicado a um material de forma a permitir o seu fluxo. Nos rejeitos este esforço aumenta exponencialmente com o aumento da concentração de sólidos (Gao e Fourie; 2015). Assim, o controle da consistência do material nas operações de disposição de rejeitos é crítica (Boger 1998; Clayton et al., 2003). Vários estudos fazem uma relação da tensão de escoamento com a concentração de sólidos dos rejeitos (Boger, 2000, 2009 e 2013; Sofrá e Boger, 2002; Gawu e Fourie; 2004).

Segundo Wei et al. (2018), a tecnologia de disposição de rejeitos enfrenta hoje grandes desafios e oportunidades para novos desenvolvimentos. O desaguamento de lamas é um processo fundamental na disposição de lamas e muito importante para uma efetiva redução dos custos finais de processamento.

A Figura 1, adaptada de Laudriault, mostra a tensão de escoamento em função da concentração de sólidos. Nesta Figura 1 são apresentados diversos aspectos relacionados ao tipo de material produzido (polpa, pasta e torta) como: velocidade de fluxo, forma de disposição, existência de segregação, tipos de equipamentos de desaguamento que podem ser utilizados, características reológicas dos fluxos e tipos de bombas para o transporte. 


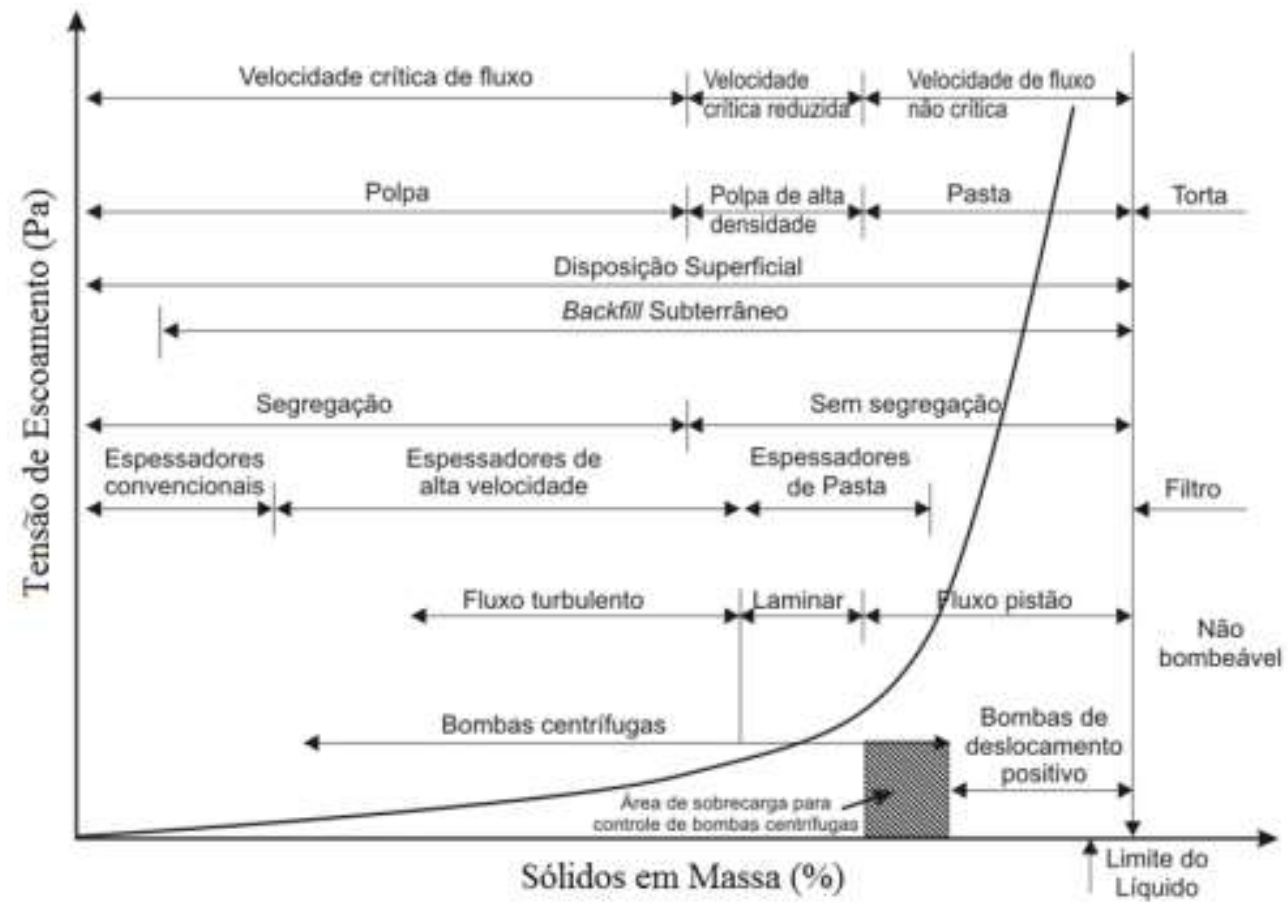

Figura 1: Tensão de escoamento em função da concentração de sólidos (Adaptado de Laudriault, 2002).

O presente trabalho tem como objetivo apresentar um comparativo de algumas das principais características de pastas minerais geradas com rejeitos provenientes do beneficiamento de minérios de manganês e fosfato visando uma possível disposição.

\section{MATERIAL E MÉTODOS}

\subsection{Materiais}

Foram utilizadas lamas de rejeitos provenientes do beneficiamento de minérios de manganês e fosfato de usinas localizadas no Brasil.

\subsection{Métodos}

Para a caracterização dos sólidos, polpas e pastas foram utilizados os métodos e equipamentos indicados na Tabela 1.

Tabela 1: Métodos e equipamentos utilizados na caracterização dos sólidos, polpas e pastas.

\begin{tabular}{lll}
\hline Propriedade & Metodologia & Equipamento \\
\hline Peso Específico & Picnometria Simples & Picnômetro \\
Granulometria & Granulômetro Difração Laser & Sympatec \\
Mineralogia & Difração Raios X & Difratômetro (Phillips, PW 3710) \\
Consistência & Teste de Abatimento & Cilindro de Policloreto de Vinila (PVC). \\
Fluidez & Teste de Calha & Calha de Acrílico. \\
Viscosidade & Viscosimetria & Viscosímetro (Brookfield, DV-III) \\
Tensão Escoamento & Vane & Reômetro (Brookfield, YR-I) \\
\hline
\end{tabular}




\subsubsection{Teste de Abatimento (Slump)}

Os testes foram realizados utilizando-se um cilindro com $10 \mathrm{~cm}$ de diâmetro e $10 \mathrm{~cm}$ de altura (NBR NM 76). A porcentagem de abatimento (\%ABT) foi calculada utilizando-se a Equação 1:

$$
\% A B T=(S / H) * 100
$$

Onde, $\mathrm{H}$ : altura do cilindro utilizada no teste e S: altura da amostra ensaiada. A Figura 2 apresenta a sequência do procedimento em laboratório do teste de abatimento.

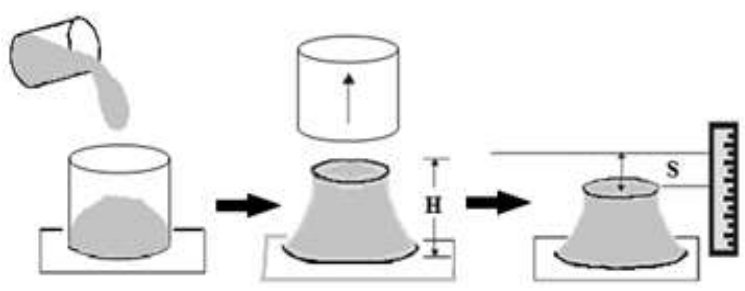

Figura 2: Teste de abatimento (Adaptado de Clayton et al, 2003).

\subsubsection{Teste de Calha}

Foi utilizada uma calha em acrílico com diferentes inclinações (0\%, 3\% e 6\%), as dimensões do equipamento mencionado podem ser observadas na Figura 3.

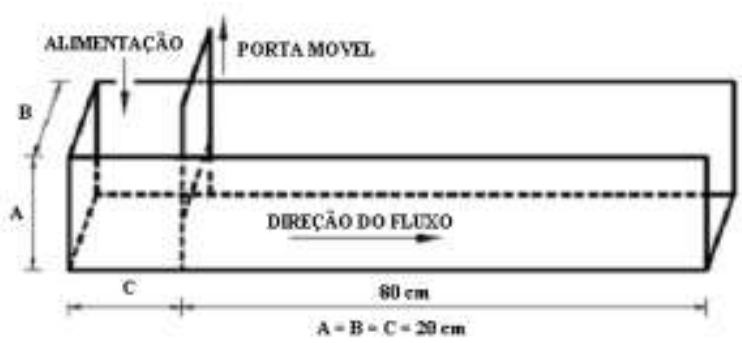

Figura 3: Teste de calha (Kwak et al, 2005).

A Equação 2 apresenta a determinação do ângulo de repouso $\left(\theta_{R}\right)$ das amostras minerais ensaiadas.

$$
\theta_{\mathrm{R}}=\operatorname{Arctg}\left[\left(h_{1}-h_{2}\right) / L\right]
$$

Onde, $h_{1}$ : altura da amostra no ponto inicial da calha; $h_{2}$ : altura da amostra no ponto final da calha; L: comprimento da amostra ao longo da calha. 


\subsubsection{Viscosimetria}

O viscosímetro digital Brookfield que dispõe de interface com o computador foi utilizado nos testes para determinação da viscosidade das amostras minerais. $O$ equipamento apresentado na Figura 4 permitiu determinar as viscosidades para diferentes concentrações de sólidos.

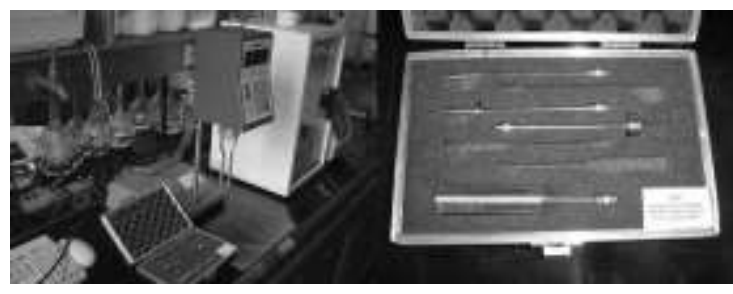

Figura 4: Viscosímetro Brookfield (modelo DV-III).

\subsubsection{Tensão de Escoamento}

Os testes foram realizados no reômetro digital Brookfield. Foi utilizado o método de vane que consiste basicamente em utilizar uma haste de quatro pás que é imersa em um recipiente contendo a amostra até uma determinada profundidade para gerar a leitura dos dados.

\section{RESULTADOS E DISCUSSÕES}

\subsection{Caracterização dos Sólidos}

A Tabela 2 mostra os resultados obtidos na caracterização dos sólidos dos rejeitos: tamanho médio $\left(d_{50}\right)$, porcentagem de material menor a $20 \mu \mathrm{m}$, peso específico e principais espécies mineralógicas.

Tabela 2: Características físicas e químicas da amostra dos rejeitos de manganês e fosfato.

\begin{tabular}{ccccc}
\hline Amostra & $\mathbf{d}_{50}(\boldsymbol{\mu m})$ & $\%<\mathbf{2 0}(\boldsymbol{\mu m})$ & Peso Específico $\left(\mathrm{g} / \mathbf{c m}^{\mathbf{3}}\right)$ & Principais Espécies \\
\hline Rejeito de Manganês & 6,23 & 85 & 2,87 & Pirolusita e Caulinita \\
Rejeito de Fosfato & 4,76 & 94 & 2,61 & Calcita e Dolomita \\
\hline
\end{tabular}

Estes resultados indicam que as amostras são muito finas, o rejeito de manganês possui uma granulometria um pouco mais grosseira e maior peso específico que o rejeito do fosfato possivelmente devido a seu conteúdo metálico. Verifica-se também que os minerais pirolusita e caulinita são predominantes no rejeito de manganês; calcita e dolomita são as espécies predominantes no rejeito de fosfato.

\subsection{Abatimento}

A Figura 5 mostra os resultados dos testes de abatimento em função das concentrações de sólidos dos rejeitos de manganês e fosfato. 

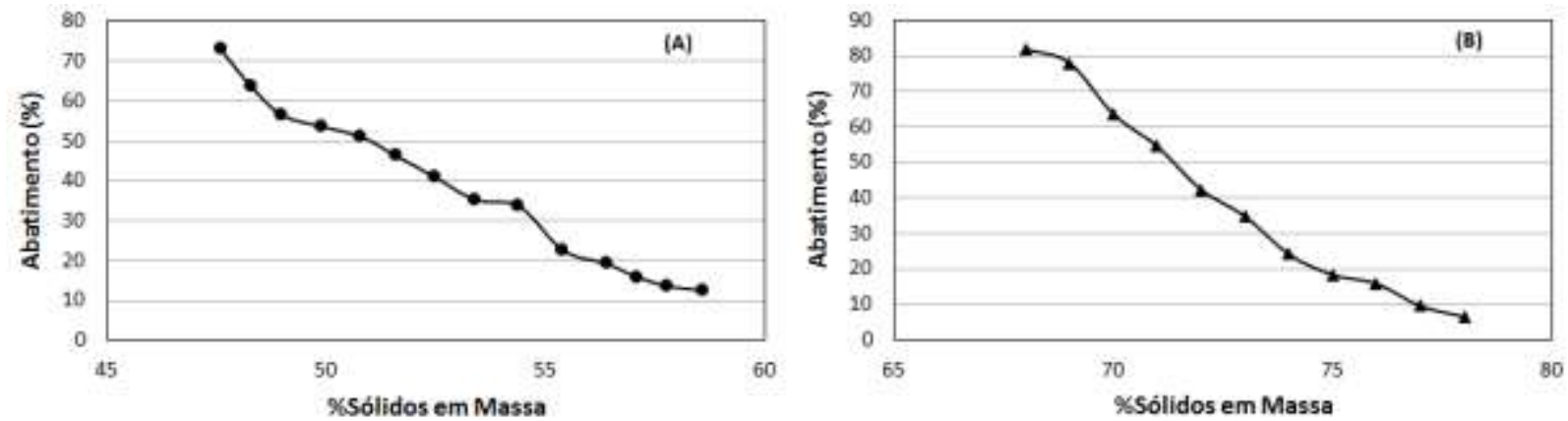

Figura 5: Abatimento (\%) versus concentração de sólidos do rejeito de manganês (A) e fosfato (B).

Observa-se pela Figura 5 que o rejeito de manganês e de fosfato se apresentam como pasta mineral numa faixa aproximada de valores entre $50-55 \%$ e $70-75 \%$ de sólidos em massa respectivamente. Os resultados indicam uma diferença significativa de comportamento dos dois materiais. Considerando-se uma possível disposição, o rejeito de fosfato apresenta uma característica mais favorável no que diz respeito a perda de água no material a ser disposto.

\section{3 Ângulo de Repouso}

A Figura 6 apresenta uma estimativa preliminar dos ângulos de repouso considerando inclinações da calha de 0\%, 3\% e 6\% em relação à horizontal em função da concentração de sólidos.
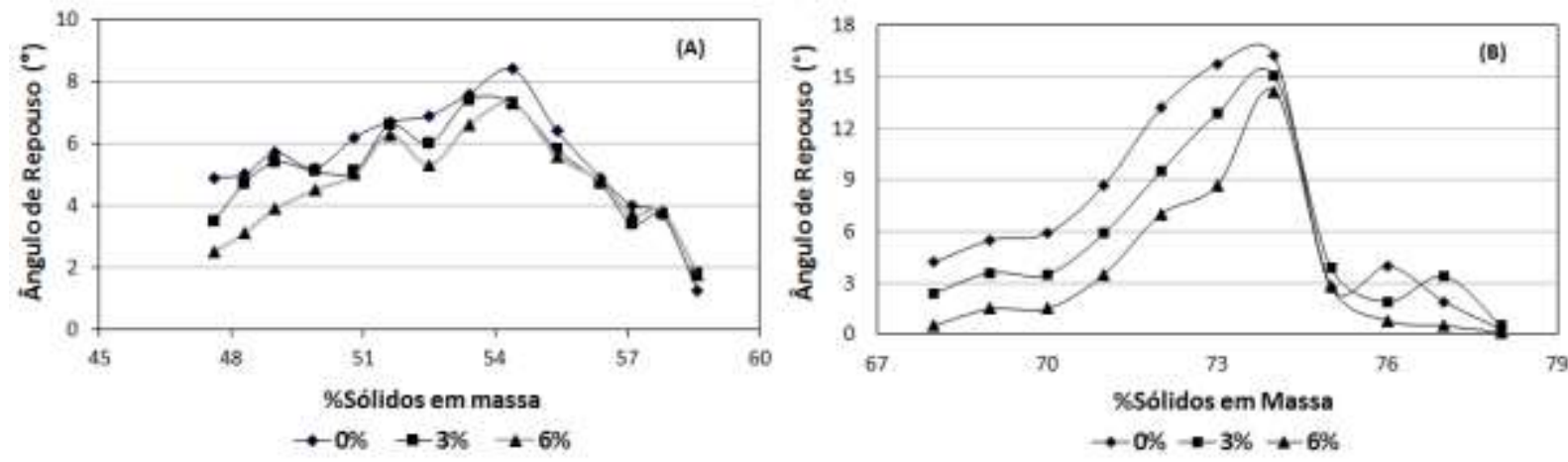

Figura 6: Ângulos de repouso em função da concentração de sólidos do rejeito de manganês (A) e fosfato (B).

A Figura 6 mostra que as melhores condições foram $54 \%$ de sólidos para o rejeito de manganês e $74 \%$ de sólidos para o rejeito de fosfato. Os ângulos obtidos com o rejeito de fosfato foram praticamente o dobro daqueles obtidos para o rejeito de manganês.

Considerando-se ainda, para efeito de comparação, que a disposição dos rejeitos fosse realizada na forma cônica e considerando-se $1^{\circ}$ o ângulo de repouso para a disposição das polpas no sistema convencional (barragem), os rejeitos de manganês poderiam ocupar um volume cerca de 9 vezes maior enquanto os rejeitos de fosfato 18 vezes maior, para uma mesma área de superfície. Não foi considerada, neste caso, a influência do fator climático. 


\subsection{Viscosidade}

A Figura 7 mostra os resultados da viscosidade dos rejeitos de manganês e fosfato para diferentes concentrações de sólidos com uma rotação fixa da haste de 30rpm.
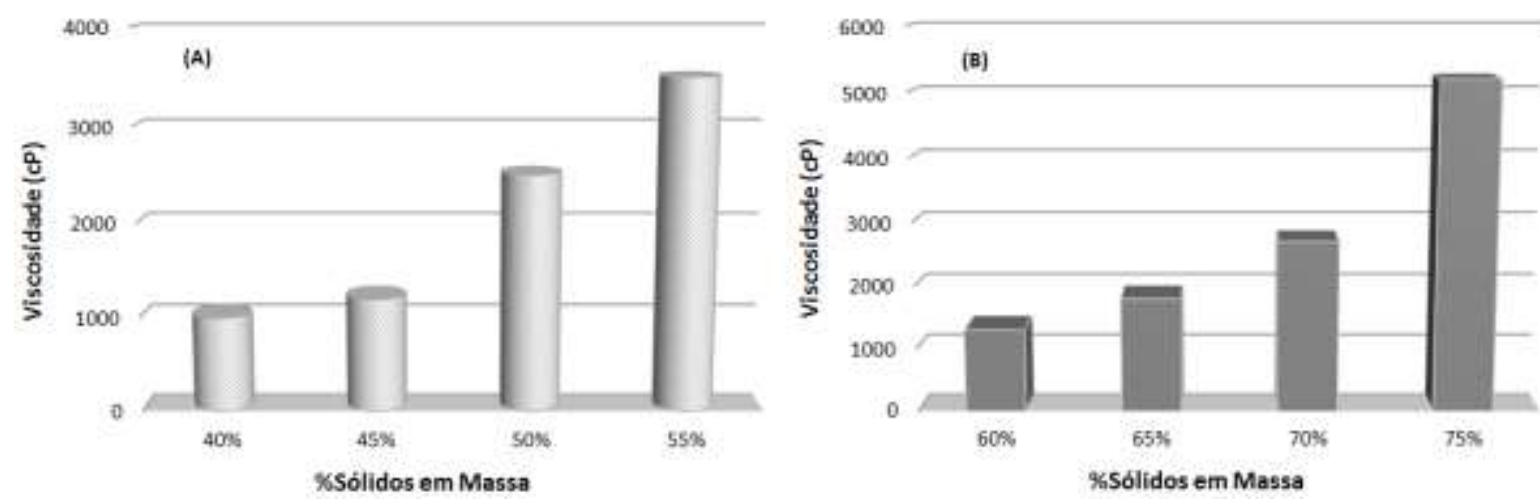

Figura 7: Viscosidade em função da concentração de sólidos do rejeito de manganês (A) e fosfato (B).

Verifica-se pela Figura 7 que a viscosidade do rejeito de fosfato foi maior que a do rejeito de manganês na faixa em que se apresentam como pasta mineral. A viscosidade do rejeito de fosfato para $75 \%$ de sólidos em massa foi de aproximadamente 5200cP e para o rejeito de manganês de 3400cP com 55\% sólidos em massa possivelmente devido a que o rejeito de fosfato possui uma maior quantidade de partículas finas $(94 \%<20 \mu \mathrm{m})$ e menor porcentagem de água. Cabe destacar que o rejeito de fosfato para $55 \%$ de sólidos em massa apresenta comportamento de polpa com diferença significativa de comportamento do rejeito de manganês nesta mesma concentração de sólidos.

\subsection{Tensão de Escoamento}

A Figura 8 apresenta as tensões de escoamento dos rejeitos de manganês e fosfato obtidas para diferentes concentrações de sólidos.
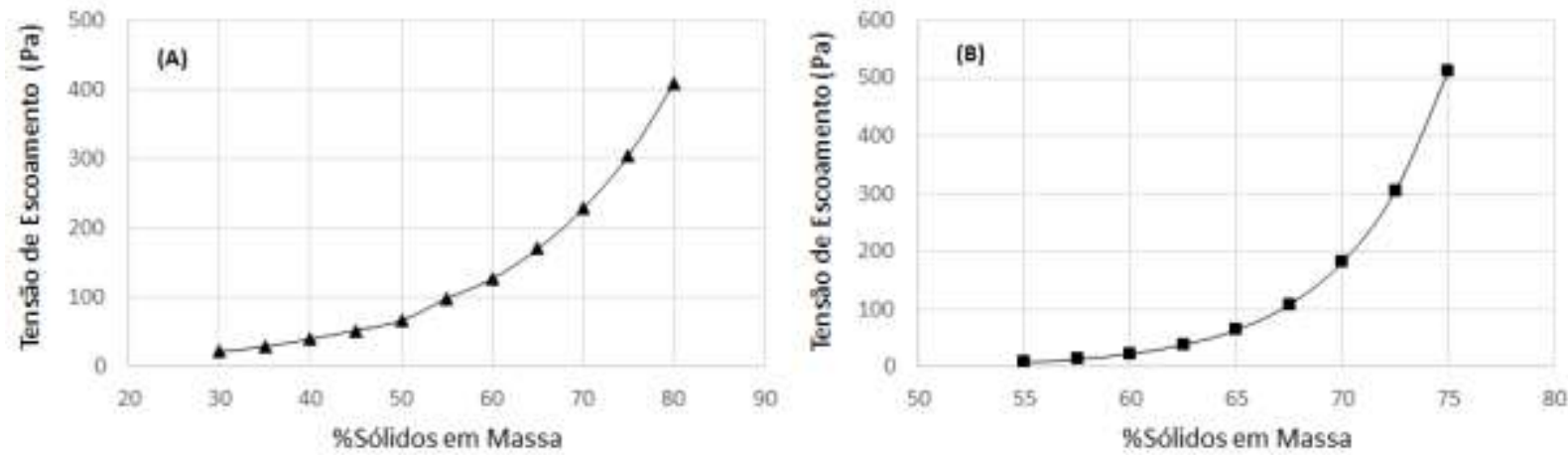

Figura 8: Tensão de escoamento em função da concentração de sólidos rejeito de manganês (A) e fosfato (B).

De acordo com a Figura 8, o valor da tensão de escoamento considerando um abatimento de $20 \%$ nas pastas minerais é de $100 \mathrm{~Pa}$ no rejeito de manganês enquanto que para o rejeito de fosfato foi de 500Pa, o que representa um incremento de 5 vezes devido provavelmente á menor quantidade de água retida. A presença de argilas no rejeito de manganês interfere no comportamento da viscosidade e da tensão de escoamento. Neste caso, as condições de bombeamento das pastas devem ser diferentes, ou seja, o rejeito de manganês poderia, em 
princípio, ser transportado por bombas centrífugas enquanto o rejeito de fosfato por bombas de deslocamento positivo. A análise de custos de investimento, custos de operação, e limitações técnicas vai definir a melhor forma de transporte e disposição das pastas.

\section{CONCLUSÕES}

$\mathrm{Na}$ caracterização do sólido dos rejeitos de manganês e fosfato se observa uma granulometria muito fina típica das lamas, fator importante na formação de pastas minerais. Os minerais pirolusita e caulinita são predominantes no rejeito de manganês; calcita e dolomita são as espécies predominantes no rejeito de fosfato. Os testes de abatimento indicaram que numa faixa aproximada entre $50-55 \%$ de sólidos em massa para o rejeito de manganês e $70-75 \%$ de sólidos em massa para o rejeito de fosfato são obtidas pastas minerais. Os testes de calha indicaram, numa avaliação preliminar, que nas melhores condições o ângulo de repouso para o rejeito de manganês seria superior a $8^{\circ}$ e para o rejeito de fosfato de $16^{\circ}$. As características reológicas das polpas formadas pelos rejeitos de manganês e fosfato mostraram-se distintas. A viscosidade obtida para o rejeito de fosfato foi maior que a obtida para o rejeito de manganês na faixa aproximada em que se apresentam como pasta; a viscosidade do rejeito de fosfato para $75 \%$ de sólidos em massa foi de aproximadamente $5200 \mathrm{cP}$, e para o rejeito de manganês de 3400cP com $55 \%$ sólidos em massa. De forma geral, estes rejeitos estudados apresentam características distintas que devem ser levadas em conta para uma possível disposição.

\section{REFERÊNCIAS BIBLIOGRÁFICAS}

Boger D.V. (1998). Environmental rheology and the mining industry. Proceedings of the VI International Symposium on Mining with Backfill. The Australasian Institute of Mining and Metallurgy Publication Series, Brisbane, Austrália, p. 15-17.

Boger D.V. (2000). Rheology and the minerals industry. Minerals Processing Extractive Metallurgy, Rev. 20, p. 1-25.

Boger D.V. (2009). Rheology and the resource industries. Chemical Engineering Science, Vol. 64, p. $4525-4536$.

Boger D.V. (2013). Rheology of slurries and environmental impacts in the mining industry. Annual review of chemical and biomolecular engineering, Vol. 4, p. 239-257.

Clayton S., Grice T.G., Boger D.V. (2003). Analysis of the slump test for on-site yield stress measurement of mineral suspensions. International Journal of Mineral Processing, Vol. 70, p. 3-21.

Fernandez A.I., Araujo A.C., Goosens M. (2013, Jun. 17-20). Studying feasibility of paste and thickened tailings at laboratory scale - pilot thickeners. In: 16th International Seminar on Paste and Thickened Tailings; Belo Horizonte, Brazil. p. 635-650. 
Gao J., Fourie A. (2015). Using the flume tests for yield stress measurement of thickened tailings. Elsevier, Minerals Engineering, Elsevier, Vol. 81, p. 116-127.

Gawu S.K., Fourie A. (2004). Assessment of the modified slump test as a measure of the yield stress of high-density thickened tailings. Can. Geotech, Vol. 41, p. 39-47.

Kwak M., James D.F., Klein, K.A. (2005). Flow Behavior of Tailings Paste for Surface Disposal. International Journal of Mineral Processing, Toronto-Canadá, p. 1-15.

Laudriault D. (2002). The Surface Disposal of Industrial Waste: Slurry Placement v/s High Density Slurry Placement v/s Placement as a Paste. High density \& Paste Seminar, Apresentações digitais, Santiago, Chile.

Olcay R.H.B. (2012). Desenvolvimento de equipamento produtor de pasta mineral [Dissertação de Mestrado]/ Desenvolvimento de equipamento produtor de pasta mineral para aproveitamento dos rejeitos das lamas calcárias e diminuição do impacto ambiental [Teste de Doutorado]. Programa de Pós-Graduação em Engenharia Metalúrgica, Materiais e de Minas da Universidade Federal de Minas Gerais, Belo Horizonte; 2008.

Paterson A.J.C. (2004). High density slurry and paste tailings, transport systems. International Platinum Conference 'Platinum Adding Value', The South African Institute of Mining and Metallurgy, p. 159-165.

Qi Y., Thapa K., Hoadley A. (2011). Application of filtration aids for improving sludge dewatering properties - A review, Chemical Engineering Journal, Elsevier, Vol. 171, p. 373-384.

Sofrá F., Boger D.V. (2002). Environmental rheology for waste minimisation in the minerals industry. Chemical Engineering, Vol. 86, p. 319-330.

Summerhaysa R., Gaspar A. (2016). Thickening, Filtration and Clarification in the Phosphoric Acid Industry, Symphos 2015, $3^{\text {rd }}$ International Symposium on Innovation and Technology in the Phosphate Industry, ScienceDirect, Elsevier, Procedia Engineering, Vol. 138, p. 164-173.

Wei H., Gao B., Ren J., Li A., Yang H. (2018). Coagulation/flocculation in dewatering of sludge: A review, Water Research, Elsevier, Vol. 143, p. 608-631. 